\title{
Analysis of Photonic-Crystal Problems with MLFMA and Approximate Schur Preconditioners
}

\author{
Özgür Ergül ${ }^{1,2}$, Tahir Malas ${ }^{1,2}$, Seçil Kılınç ${ }^{1,2}$, Serkan Sarıtaş² ${ }^{2}$ and Levent Gürel ${ }^{1,2}$ \\ ${ }^{1}$ Department of Electrical and Electronics Engineering \\ ${ }^{2}$ Computational Electromagnetics Research Center (BiLCEM) \\ Bilkent University, TR-06800, Bilkent, Ankara, Turkey \\ lgurel@ee.bilkent.edu.tr
}

\begin{abstract}
We consider fast and accurate solutions of electromagnetics problems involving three-dimensional photonic crystals (PhCs). Problems are formulated with the combined tangential formulation (CTF) and the electric and magnetic current combined-field integral equation (JMCFIE) discretized with the Rao-Wilton-Glisson functions. Matrix equations are solved iteratively by the multilevel fast multipole algorithm. Since $\mathbf{P h C}$ problems are difficult to solve iteratively, robust preconditioning techniques are required to accelerate iterative solutions. We show that novel approximate Schur preconditioners enable efficient solutions of $\mathrm{PhC}$ problems by reducing the number of iterations significantly for both CTF and JMCFIE.
\end{abstract}

\section{INTRODUCTION}

Photonic crystals (PhCs) are artificial structures, which are usually constructed by periodically arranging dielectric unit cells, such as periodic slabs and a perforated $\mathrm{PhC}$ waveguide depicted in Fig. 1. Those structures exhibit frequency-selective electromagnetic responses, i.e., their electromagnetic transmission properties change rapidly as a function of frequency. For example, the $\mathrm{PhC}$ structure involving periodic rectangular slabs in Fig. 1(a) is usually transparent, but it becomes opaque and inhibits the transmission of electromagnetic waves in some frequency bands [1]. This structure can be used as a filter in microwave circuits and antenna systems. The perforated $\mathrm{PhC}$ structure in Fig. 1(b) is also frequency-selective, and it can be used as an efficient waveguide to change the direction of electromagnetic waves [2].

In this study, we consider fast and accurate solutions of electromagnetics problems involving three-dimensional $\mathrm{PhCs}$, such as depicted in Fig. 1. Problems are formulated with the combined tangential formulation (CTF) [3] and the electric and magnetic current combined-field integral equation (JMCFIE) [4], discretized with the Rao-Wilton-Glisson (RWG) functions [5]. Matrix equations are solved iteratively using the multilevel fast multipole algorithm (MLFMA) [6], and iterative solutions are accelerated via novel approximate Schur preconditioners (ASPs). We extensively investigate solutions of PhC problems in terms of accuracy and efficiency. We show that ASPs reduce the number of iterations significantly for both CTF and JMCFIE.

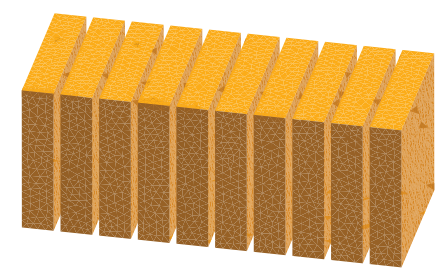

(a)

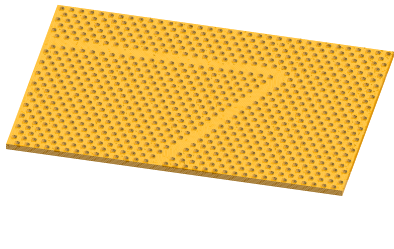

(b)
Fig. 1. Two types of $\mathrm{PhC}$ problems considered in this paper; (a) periodic slabs and (b) perforated $\mathrm{PhC}$ waveguide.

\section{Surface Formulations FOR Dielectric Problems}

In the literature, various dielectric formulations are available for the solution of dielectric problems. Among many choices, CTF and JMCFIE are usually most suitable formulations in terms of accuracy and efficiency [7]. CTF is a modified and more stable version of the well-known Poggio-MillerChang-Harrington-Wu-Tsai (PMCHWT) formulation [3]. This formulation is practically a first-kind integral equation, and it produces ill-conditioned matrix equations without preconditioning. Nevertheless, accuracy of CTF is excellent, even when it is discretized with low-order basis functions, such as the RWG functions. On the other hand, JMCFIE is a second-kind integral equation and it usually produces betterconditioned matrix equations compared to CTF. Unfortunately, the accuracy of JMCFIE can be poor, especially when it is discretized with low-order basis functions, due to the excessive discretization error of the identity operator [8]. In addition, the accuracy of JMCFIE further deteriorates as the contrast of the object increases and/or the object involves sharp edges and corners [9].

Discretizations of integral-equation formulations for homogeneous objects lead to $2 N \times 2 N$ dense matrix equations in the form of

$$
\left[\begin{array}{ll}
\overline{\boldsymbol{Z}}_{11} & \overline{\boldsymbol{Z}}_{12} \\
\overline{\boldsymbol{Z}}_{21} & \overline{\boldsymbol{Z}}_{22}
\end{array}\right] \cdot\left[\begin{array}{l}
\boldsymbol{a}_{J} \\
\boldsymbol{a}_{M}
\end{array}\right]=\left[\begin{array}{l}
\boldsymbol{v}_{1} \\
\boldsymbol{v}_{2}
\end{array}\right], \text { or } \overline{\boldsymbol{Z}} \cdot \boldsymbol{a}=\boldsymbol{v},
$$

where $\overline{\boldsymbol{Z}} \in \mathbb{C}^{2 N \times 2 N}$ and $\overline{\boldsymbol{Z}}_{11}, \overline{\boldsymbol{Z}}_{12}, \overline{\boldsymbol{Z}}_{21}, \overline{\boldsymbol{Z}}_{22} \in \mathbb{C}^{N \times N}$. Solutions of (1) via Krylov-subspace algorithms provide expansion coefficients $\boldsymbol{a}_{J}$ and $\boldsymbol{a}_{M}$ for equivalent electric and 
magnetic currents, respectively. Using expansion coefficient, scattered electric and magnetic fields can be calculated everywhere.

Matrix-vector multiplications (MVMs) required by iterative algorithms can be performed efficiently with $\mathcal{O}(N \log N)$ complexity using MLFMA. This method is based on the calculation of interactions between basis and testing functions in a group-by-group manner in a multilevel scheme. In the case of dielectric problems, MLFMA must be applied for both inner and outer media [7].

\section{ApProximate SChUr Preconditioners}

Both CTF and JMCFIE lead to non-hermitian and indefinite systems, whose iterative solutions may not converge easily without preconditioning. Being a second-kind integral equation, JMCFIE leads to diagonally-dominant matrices so that simple block-diagonal preconditioners can be effective [7]. However, CTF does not provide diagonally-dominant matrices, and its efficient solutions may require strong preconditioners constructed from all available interactions in MLFMA, i.e., near-field matrices.

Preconditioning techniques for systems similar to (1) are usually studied in the context of generalized-saddle-point problems [10]-[15]. Approximating the dense matrix in (1) by a sparse near-field matrix $\bar{Z}^{N F}$, preconditioners developed for saddle-point problems can be used for integral-equation solutions of dielectric problems. In general, those preconditioners are obtained with some approximations to the Schur complement reduction, which decomposes the solution of a $2 \times 2$ partitioned near-field system

$$
\left[\begin{array}{ll}
\overline{\boldsymbol{Z}}_{11}^{N F} & \overline{\boldsymbol{Z}}_{12}^{N F} \\
\overline{\boldsymbol{Z}}_{21}^{N F} & \overline{\boldsymbol{Z}}_{22}^{N F}
\end{array}\right] \cdot\left[\begin{array}{l}
\boldsymbol{x} \\
\boldsymbol{y}
\end{array}\right]=\left[\begin{array}{l}
\boldsymbol{f} \\
\boldsymbol{g}
\end{array}\right],
$$

into solutions of two systems, i.e.,

$$
\begin{aligned}
\overline{\boldsymbol{Z}}_{11}^{N F} \cdot \boldsymbol{x} & =\boldsymbol{f}-\overline{\boldsymbol{Z}}_{12}^{N F} \cdot \boldsymbol{y} \\
\overline{\boldsymbol{S}} \cdot \boldsymbol{y} & =\boldsymbol{g}-\overline{\boldsymbol{Z}}_{21}^{N F} \cdot\left(\overline{\boldsymbol{Z}}_{11}^{N F}\right)^{-1} \cdot \boldsymbol{f},
\end{aligned}
$$

where

$$
\overline{\boldsymbol{S}}=\overline{\boldsymbol{Z}}_{22}^{N F}-\overline{\boldsymbol{Z}}_{21}^{N F} \cdot\left(\overline{\boldsymbol{Z}}_{11}^{N F}\right)^{-1} \cdot \overline{\boldsymbol{Z}}_{12}^{N F} .
$$

Success of those preconditioners depend on fast and efficient solutions of (3) and (4). Hence, we need effective approximations to the inverse of $\overline{\boldsymbol{Z}}_{11}^{N F}$, as well as the inverse of $\overline{\boldsymbol{S}}$.

One can use the sparse-approximate inverse (SAI) of $\overline{\boldsymbol{Z}}_{11}^{N F}$, i.e.,

$$
\overline{\boldsymbol{M}}_{11} \approx\left[\overline{\boldsymbol{Z}}_{11}^{N F}\right]^{-1}
$$

in (4) and (5). Then, the solution of (3) can be written as

$$
\boldsymbol{x} \approx \overline{\boldsymbol{M}}_{11} \cdot\left(\boldsymbol{f}-\overline{\boldsymbol{Z}}_{12}^{N F} \cdot \boldsymbol{y}\right) .
$$

However, a good approximation to $\boldsymbol{y}$ is required in (7). Consequently, we also need to develop a good approximation to the Schur complement matrix.

We note that, in many applications involving partitioned systems, the partition $\bar{Z}_{22}^{N F}$ is identically zero, or it consists of very small elements compared to elements in other partitions [10]. Unfortunately, this is not valid for matrix equations
TABLE I

PHC PROBLEMS

\begin{tabular}{|c|c|c|c|}
\hline \multicolumn{4}{|c|}{ PERIODIC SLABS } \\
\hline Problem & Slab Size $(\mathrm{m})$ & Number of Walls & Unknowns \\
\hline S1 & $0.41 \times 2 \times 2$ & 5 & 38,700 \\
\hline S2 & $0.41 \times 2 \times 2$ & 10 & 77,400 \\
\hline S3 & $0.41 \times 4 \times 4$ & 5 & 131,460 \\
\hline S4 & $0.41 \times 4 \times 4$ & 10 & 262,920 \\
\hline \multicolumn{4}{|c|}{ PERFORATED PhC WAVEGUIDE } \\
\hline Problem & Size $(\mathrm{cm})$ & Number of Holes & Unknowns \\
\hline P1 & $0.6 \times 5 \times 5$ & 18 & 14,226 \\
\hline P2 & $0.6 \times 5 \times 10$ & 38 & 27,798 \\
\hline P3 & $0.6 \times 15 \times 20$ & 272 & 162,420 \\
\hline P4 & $0.6 \times 26 \times 34$ & 828 & 475,782 \\
\hline
\end{tabular}

obtained from CTF and JMCFIE, and we are unable use many techniques developed for those cases in the literature. In our case, an applicable method can be using a Krylov-subspace solver to obtain an approximate solution of the system in (4). MVMs with $\bar{S}$ can be performed by approximating the inverse of $\bar{Z}_{11}^{N F}$ with $\bar{M}_{11}$. However, a robust preconditioner for $\bar{S}$ is still required.

One option is to ignore the second term in (5) and to approximate the inverse of the Schur complement by $\bar{M}_{22}$, i.e., SAI of $\overline{\boldsymbol{Z}}_{22}^{N F}$. For both CTF and JMCFIE, $\overline{\boldsymbol{Z}}_{22}^{N F}=\overline{\boldsymbol{Z}}_{11}^{N F}$, hence $\bar{M}_{11}$ can also provide an approximation to the inverse of the Schur complement $\overline{\boldsymbol{S}}$. Hence, one can find an approximation to $\boldsymbol{y}$ as

$$
\boldsymbol{y} \approx \overline{\boldsymbol{M}}_{11} \cdot\left(\boldsymbol{g}-\overline{\boldsymbol{Z}}_{21}^{N F} \cdot \overline{\boldsymbol{M}}_{11} \cdot \boldsymbol{f}\right),
$$

which can be used in (7). We call the resulting preconditioner defined by (7) and (8) as ASP. $\bar{M}_{11}$ can also be used as a preconditioner for iterative solutions of (3) and (4), provided that $\bar{M}_{11}$ is used instead of exact inverses in (5) and (4). In this case, the preconditioner is called iterative ASP (IASP). We note that a flexible solver is required for IASP since the effective preconditioner changes from iteration to iteration [16]. Finally, if $\bar{M}_{11}$ does not provide a good approximation to $\bar{S}$, a better approximation to the inverse of the Schur complement can be obtained via incomplete MVMs, as detailed in [17].

\section{RESULTS}

Table I lists $\mathrm{PhC}$ problems involving periodic slabs and perforated $\mathrm{PhC}$ waveguides considered in this paper. All structures are located in free space and illuminated by a Hertzian dipole. The relative permittivity of $\mathrm{PhCs}$ involving periodic slabs is 4.8, and they are expected to resonate at $300 \mathrm{MHz}$. Those structures are investigated at $250 \mathrm{MHz}, 300 \mathrm{MHz}$, and $350 \mathrm{MHz}$. Perforated $\mathrm{PhC}$ waveguides have relative permittivities of 12.0 and they are investigated at $8.25 \mathrm{GHz}$, i.e., at the frequency for the most efficient transmission. Problems are formulated with both CTF and JMCFIE. Periodic slabs are discretized with $\lambda / 10$ triangles, where $\lambda$ is the wavelength in free space. Perforated $\mathrm{PhC}$ slabs require finer triangulations with $\lambda / 20$ triangles for accurate modelling of air holes. 

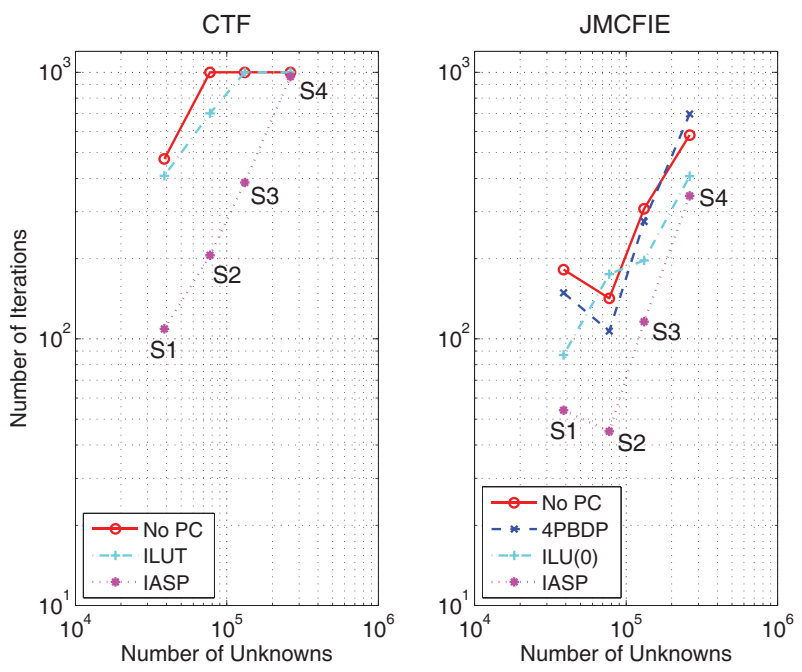

Fig. 2. Number of iterations for the solution of periodic-slab problems listed in Table I.

Iterative solutions are performed by the generalized-minimalresidual (GMRES) method [16] without a restart. The relative residual error for the convergence is set to $10^{-3}$. Iterations are started with a zero initial guess and terminated at maximum 1000th iteration.

Fig. 2 depicts the solution of periodic-slab problems at $300 \mathrm{MHz}$. We compare iteration counts when solutions are accelerated with a four-partition block-diagonal preconditioner (4PBDP) [7], ILU-type preconditioners (ILU(0) for JMCFIE and ILUT for CTF) [16]), and IASP, in addition to the no-preconditioner (NP) case. Solutions employing 4PBDP are omitted for CTF since they do not converge. In addition, CTF solutions do not converge for large problems without preconditioning or when using ILU(0). In the case of IASP, we use only $\bar{M}_{11}$ as the inner preconditioner so that the amount of memory required by this preconditioner is $1 / 4$ of that of ILU-type preconditioners. Fig. 2 shows that IASP provides the most efficient solutions of periodic-slab problems.

Fig. 3 depicts the solution of perforated-PhC problems. In this case, we omit ILU-type preconditioners since their memory requirement is excessively large for the largest two problems. In addition, 4PBDP is again omitted for CTF due to nonconvergent solutions. Fig. 3 shows that ASP, which employs incomplete MVMs, significantly accelerates iterative solutions of CTF. ASP is also very effective for JMCFIE and reduces the number of iterations significantly, compared to solutions without preconditioning and with 4PBDP. We note that the largest problem in Fig. 3 cannot be solved without using ASP.

Fig. 4 presents power transmission results for the periodicslabs problem S2. The power transmission is calculated pointwise around dielectric slabs, and the transmission properties of the structure is investigated at $250 \mathrm{MHz}, 300 \mathrm{MHz}$, and $350 \mathrm{MHz}$. We observe that the structure is transparent at $250 \mathrm{MHz}$ and $350 \mathrm{MHz}$, i.e., the power transmission is unity in the transmission region on the left-hand side of the structure.
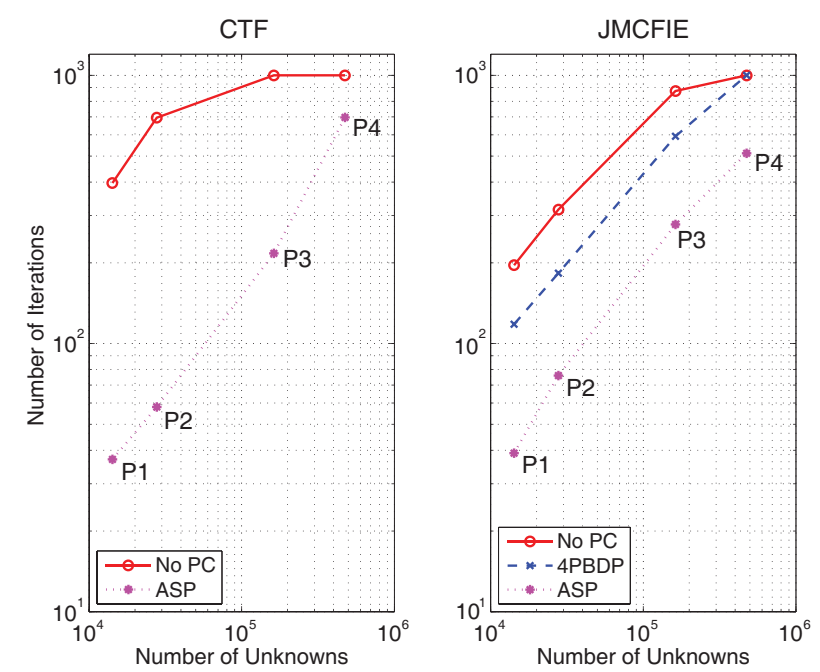

Fig. 3. Number of iterations for the solution of perforated-PhC problems listed in Table I.

At $300 \mathrm{MHz}$, however, the structure becomes opaque and a shadowing occurs. We also note that there are discrepancies between results obtained by using CTF and JMCFIE; this is mostly due to the inaccuracy of JMCFIE.

Fig. 5 presents near-zone magnetic fields for the perforated$\mathrm{PhC}$ problem $\mathrm{P} 4$. The total magnetic field is calculated pointwise inside and outside the structure in order to demonstrate the transmission of electromagnetic waves from the left-hand side to the bottom. For this problem, we observe that results obtained by using CTF and JMCFIE are significantly different. This is due to the deteriorating accuracy of JMCFIE in the case of complicated structures and relatively high contrasts.

Finally, in order to show that the inconsistency between CTF and JMCFIE results is due to the inaccuracy of JMCFIE, we consider the solution of an electromagnetics problem involving a $0.6 \mathrm{~cm} \times 7 \mathrm{~cm} \times 10 \mathrm{~cm}$ perforated $\mathrm{PhC}$ waveguide. The problem is formulated with CTF and JMCFIE discretized by using $\lambda / 20$ and $\lambda / 40$ triangles. Fig. 6 presents the magnetic field at $8.25 \mathrm{GHz}$. We observe that results obtained by JMCFIE change drastically when the discretization is refined. Specifically, JMCFIE results become consistent with CTF results for the dense discretization.

\section{CONCLUding REMARKS}

In this paper, we consider MLFMA solutions of electromagnetics problems involving three-dimensional $\mathrm{PhCs}$ formulated with CTF and JMCFIE. In addition to MLFMA, robust preconditioning techniques are required in order to solve $\mathrm{PhC}$ problems efficiently. We show that novel ASPs accelerate iterative solutions significantly and they enable the analysis of relatively large $\mathrm{PhC}$ structures.

\section{ACKNOWLEDGMENT}

This work was supported by the Turkish Academy of Sciences in the framework of the Young Scientist Award Program (LG/TUBA-GEBIP/2002-1-12), by the Scientific and 

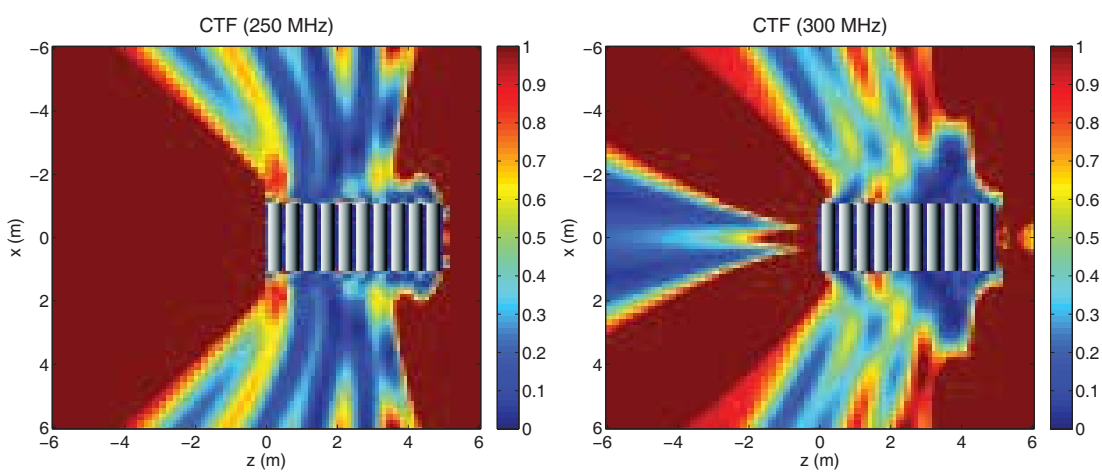

(a)
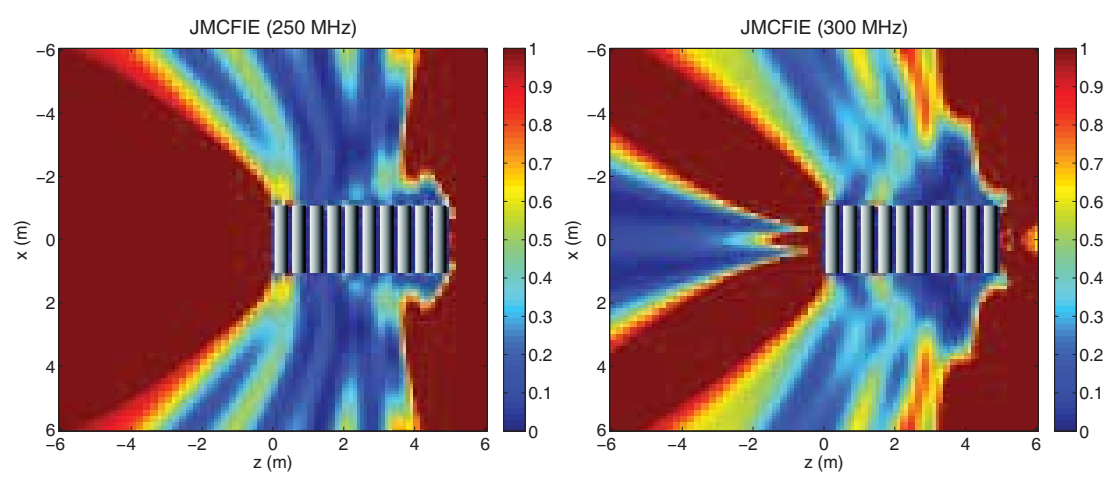

(b)
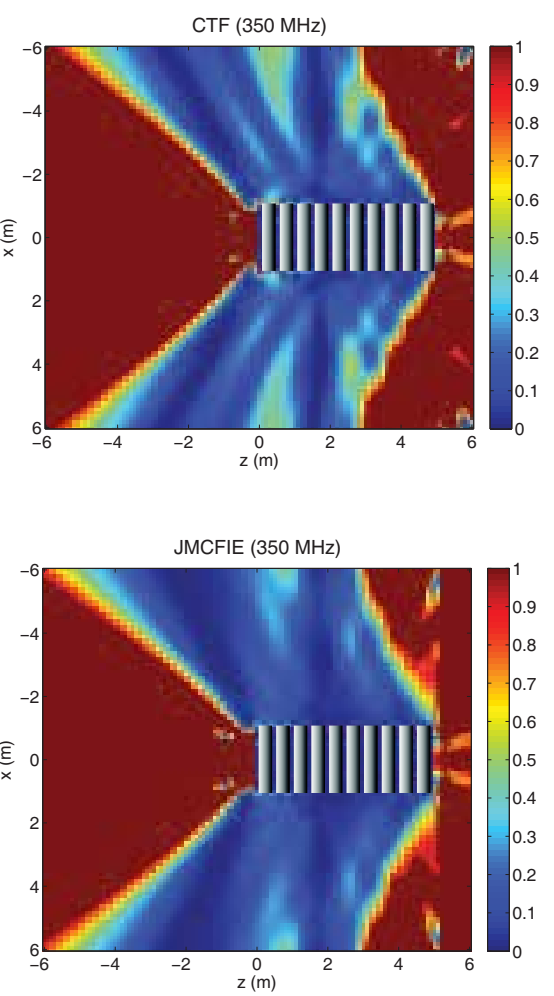

Fig. 4. Power transmission for a PhC involving periodic slabs (S2 in Table I) at $250 \mathrm{MHz}, 300 \mathrm{MHz}$, and $350 \mathrm{MHz}$, obtained by using (a) CTF and (b) JMCFIE.

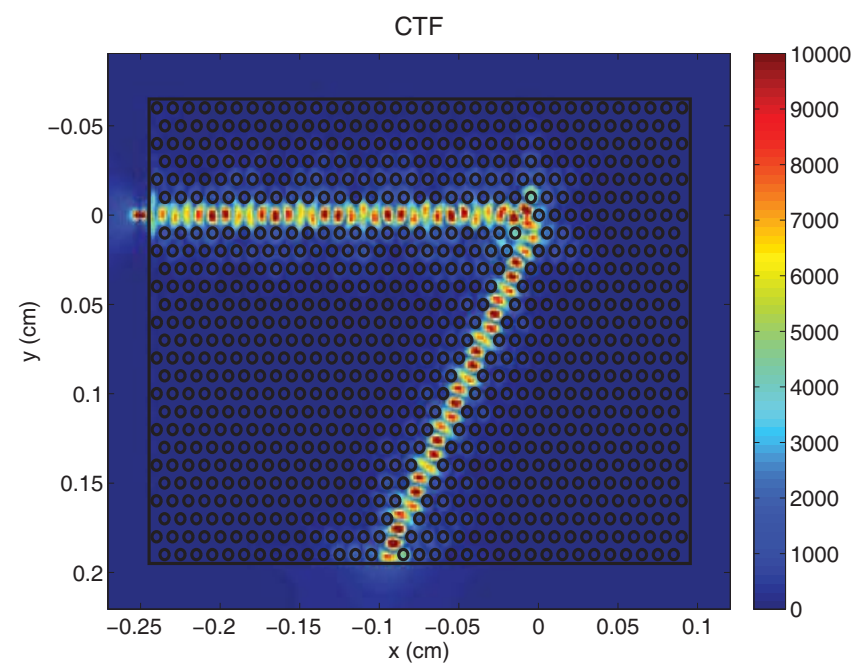

(a)

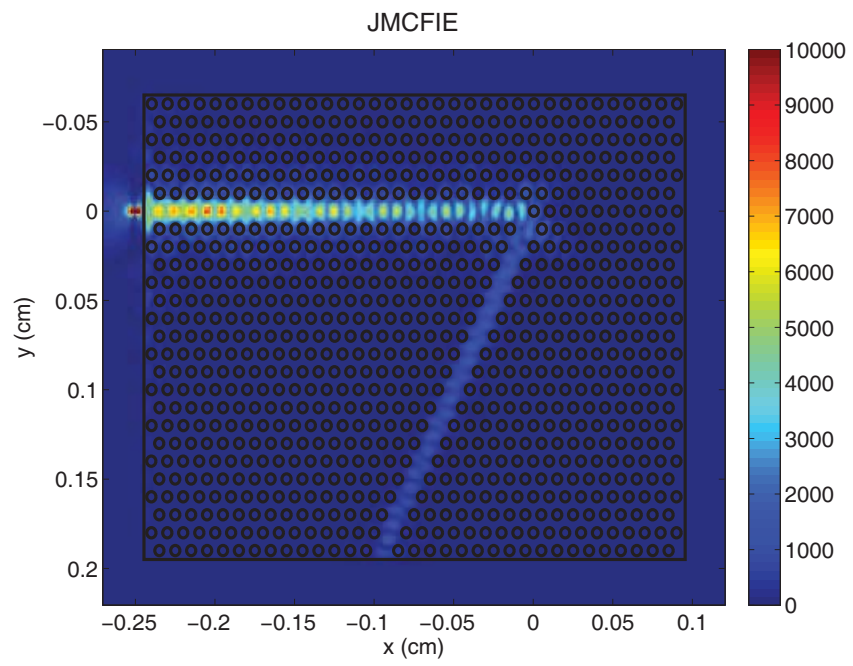

(b)

Fig. 5. Near-zone magnetic fields for a perforated $\mathrm{PhC}$ waveguide ( $\mathrm{P} 4$ in Table $\mathrm{I}$ ) illuminated by a Hertzian dipole radiating from $x=-0.25$ cm. The problem is formulated with (a) CTF and (b) JMCFIE. 




(a)

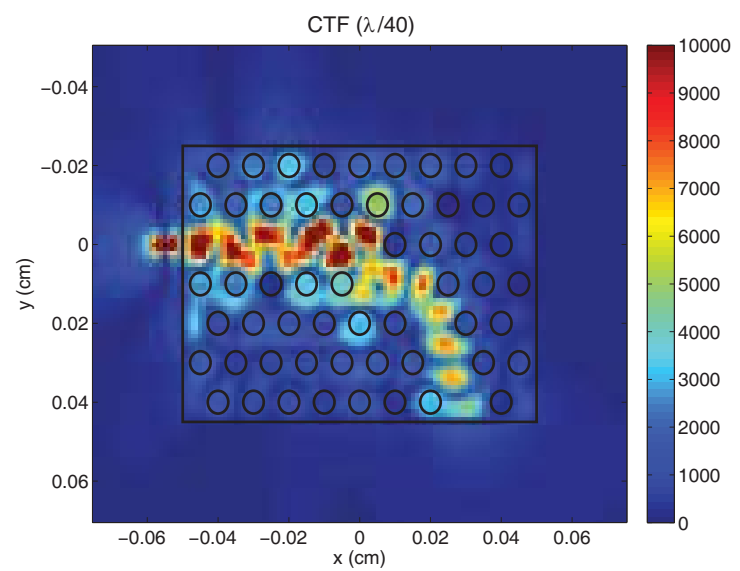

(c)

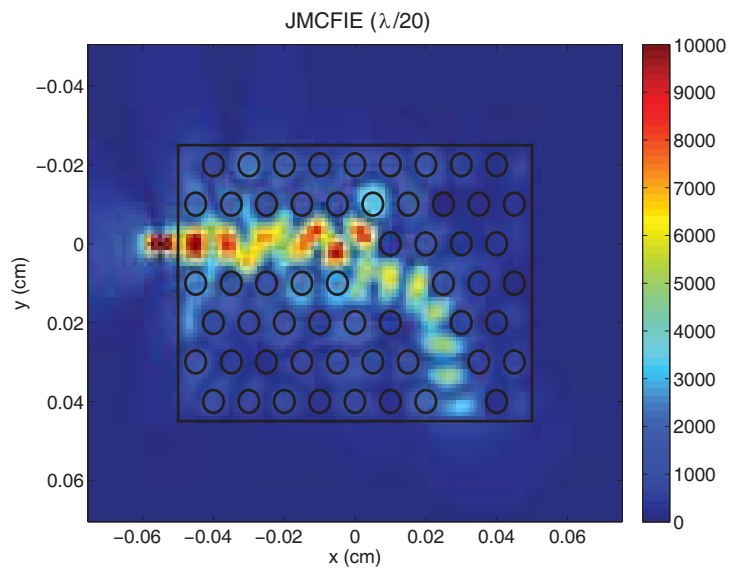

(b)

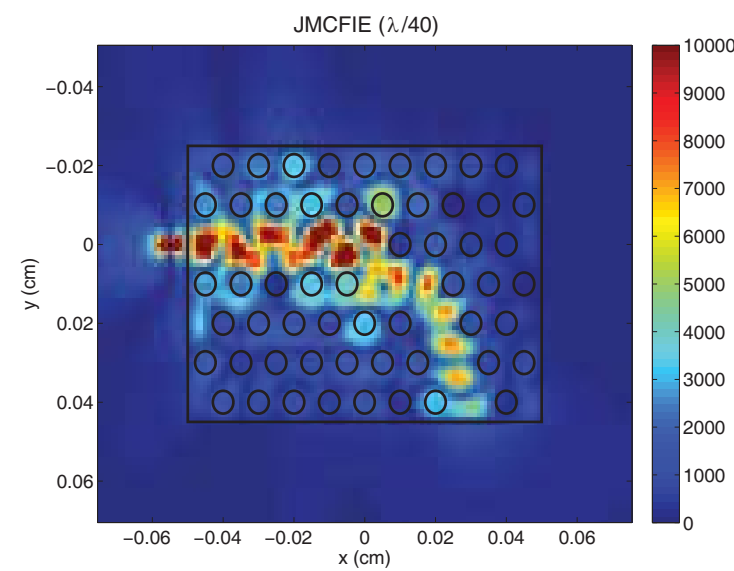

(d)

Fig. 6. Near-zone magnetic fields for a perforated $\mathrm{PhC}$ waveguide involving $7 \times 10$ holes illuminated by a Hertzian dipole. Solutions are obtained with (a) CTF and $\lambda / 20$ triangulation, (b) JMCFIE and $\lambda / 20$ triangulation, (c) CTF and $\lambda / 40$ triangulation, and (d) JMCFIE and $\lambda / 40$ triangulation.

Technical Research Council of Turkey (TUBITAK) under Research Grants 105E172 and 107E136, and by contracts from ASELSAN and SSM.

\section{REFERENCES}

[1] P. Loschialpo, D. W. Forester, and J. Schelleng, "Anomalous transmission through near unit index contrast dielectric photonic crystals," $J$. Appl. Phys., vol. 86, no. 10, pp. 5342-5347, 1999.

[2] S. G. Johnson, S. Fan, P. R. Villeneuve, and J. D. Joannopoulos, "Guided modes in photonic crystal slabs," Physical Review B, vol. 60, no. 8, pp. 5751-5758, 1999.

[3] P. Ylä-Oijala, M. Taskinen, and S. Järvenpää, "Surface integral equation formulations for solving electromagnetic scattering problems with iterative methods," Radio Science, vol. 40, RS6002, doi:10.1029/2004RS003169, no. 6, 2005.

[4] P. Ylä-Oijala and M. Taskinen, "Application of combined field integral equation for electromagnetic scattering by composite metallic and dielectric objects," IEEE Trans. Antennas Propagat., vol. 53, no. 3, pp. $1168-1173,2005$.

[5] S. Rao, D. R. Wilton, and A. W. Glisson, "Electromagnetic scattering by surfaces of arbitrary shape," IEEE Trans. Antennas Propogat., vol. AP-30, pp. 409-418, 1982.

[6] W. C. Chew, J.-M. Jin, E. Michielssen, and J. Song, Eds., Fast and Efficient Algorithms in Computational Electromagnetics. Norwood, MA, USA: Artech House, Inc., 2001.

[7] Ö. Ergül and L. Gürel, "Comparison of integral-equation formulations for the fast and accurate solution of scattering problems involving dielectric objects with multilevel fast multipole algorithm," IEEE Trans. Antennas Propagat., vol. 57, no. 1, pp. 176-187, Jan. 2009.

[8] — , "Discretization error due to the identity operator in surface integral equations," Comput. Phys. Comm., in press, 2009.

[9] P. Ylä-Oijala, M. Taskinen, and S. Järvenpää, "Analysis of surface integral equations in electromagnetic scattering and radiation problems," Eng. Anal. Boundary Elem., vol. 32, no. 3, pp. 196-209, 2008.

[10] M. Benzi, G. H. Golub, and J. Liesen, "Numerical solution of saddle point problems," Acta Numer., vol. 14, pp. 1-137, 2005.

[11] E. Chow and Y. Saad, "Approximate inverse techniques for blockpartitioned matrices," SIAM J. Sci. Comput., vol. 18, no. 6, pp. 16571675, 1997.

[12] C. Siefert and E. de Sturler, "Preconditioners for generalized saddlepoint problems," SIAM J. Numer. Anal., vol. 44, no. 3, pp. 1275-1296, 2006.

[13] M. Benzi and G. H. Golub, "A preconditioner for generalized saddle point problems," SIAM Journal on Matrix Analysis and Applications, vol. 26 , no. 1 , pp. 20-41, 2005.

[14] I. C. F. IPSEN, "A note on preconditioning nonsymmetric matrices," SIAM J. Sci. Comput., vol. 23, no. 3, pp. 1050-1051, 2002.

[15] G. Schmidlin, U. Fischer, Z. Andjeliĉ, and C. Schwab, "Preconditioning of the second-kind boundary integral equations for $3 \mathrm{D}$ eddy current problems," Int. J. Numer. Methods Eng., vol. 51, no. 9, 2001.

[16] Y. Saad, Iterative Methods for Sparse Linear Systems, 2nd ed. Philadelphia: SIAM, 2003.

[17] T. Malas and L. Gürel, "Increasing robustness and efficiency of surfaceintegral-equation solutions of dielectric problems with approximate schur preconditioners," in 2009 Computational Electromagnetics International Workshop (CEM'09), Izmir, Turkey, July 2009. 